\title{
ON EXTREMALS FOR THE TRUDINGER-MOSER INEQUALITY WITH VANISHING WEIGHT IN THE N-DIMENSIONAL UNIT BALL
}

\author{
MENGJIE ZHANG
}

Abstract. In this paper, we study the extremal function for the Trudinger-Moser inequality with vanishing weight in the unit ball $\mathbb{B} \subset \mathbb{R}^{N}(N \geqslant 3)$. To be exact, let $\mathscr{S}$ be the set of all decreasing radially symmetrical functions and $\alpha_{N}=N \omega_{N-1}^{1 /(N-1)}$, where $\omega_{N-1}$ is the area of the unit sphere in $\mathbb{R}^{N}$. Suppose $h$ is a nonnegative radially symmetrical function belonging to $C^{0}(\overline{\mathbb{B}})$ satisfying $h(x)>0$ in $\overline{\mathbb{B}} \backslash\{0\}$ and $h(x)|x|^{-N \beta} \rightarrow 1$ as $x \rightarrow 0$ for some real number $\beta \geqslant 0$. By means of blow-up analysis, we prove that the supremum

$$
\Lambda_{\beta}:=\sup _{u \in W_{0}^{1, N}(\mathbb{B}) \cap \mathscr{S},\|\nabla u\|_{N} \leqslant 1} \int_{\mathbb{B}} \exp \left\{\alpha_{N}(1+\beta)|u|^{\frac{N}{N-1}}\right\} h(x) d x
$$

can be attained by some $u_{0} \in W_{0}^{1, N}(\mathbb{B}) \cap \mathscr{S}$ with $\left\|\nabla u_{0}\right\|_{N}=1$. This improves a recent result of Yang-Zhu [39].

Mathematics subject classification (2010): 35J15, 46E35.

Keywords and phrases: Trudinger-Moser inequality, blow-up analysis, extremal function.

\section{REFERENCES}

[1] Adimurthi And O. DRUET, Blow-up analysis in dimension 2 and a sharp form of Trudinger-Moser inequality, Comm. Partial Differential Equations, 29, (2004) 295-322.

[2] Adimurthi And K. SAndeEP, A singular Moser-Trudinger embedding and its applications, Nonlinear Differ. Equ. Appl., 13, (2007) 585-603.

[3] Adimurthi AND Y. YAng, An interpolation of Hardy inequality and Trudinger-Moser inequality in $R^{N}$ and its applications, Int. Math. Res. Notices, 13, (2010) 2394-2426.

[4] D. Bonheure, E. Serra And M. TARAllo, Symmetry of extremal functions in Moser-Trudinger inequalities and a Hénon type problem in dimension two, Adv. Differential Equations, 13, (2008) 105-138.

[5] M. Calanchi And E. TerRAneO, Non-radial maximizers for functionals with exponential nonlinearity in $R^{2}$, Adv. Nonlinear Stud., 5, (2005) 337-350.

[6] L. CARLESON AND A. CHANG, On the existence of an extremal function for an inequality of J. Moser, Bull. Sci. Math., 110, (1986) 113-127.

[7] G. Csato AND P. Roy, Extremal functions for the singular Moser-Trudinger inequality in 2 dimensions, Calc. Var., 54, (2015) 2341-2366.

[8] R. Dalmasso, Problème de Dirichlet homogène pour une équation biharmonique semi-linéaire dans une boule, Bull. Sci. Math., 114, (1990) 123-137.

[9] D. De Figueiredo, E. Dos SAntos And O. MiyagaKi, Sobolev spaces of symmetric functions and applications, J. Funct. Anal., 261, (2011) 3735-3770.

[10] D. DE Figueiredo, J. Do Ó AND E. Dos SAnTos, Trudinger-Moser inequalities involving fast growth and weights with strong vanishing at zero, Proc. Amer. Math. Soc., 144, (2016) 3369-3380.

[11] W. Ding, J. Jost, J. Li And G. WANG, The differential equation $\Delta u=8 \pi-8 \pi$ he $u$ on a compact Riemann Surface, Asian J. Math., 1, (1997) 230-248.

[12] J. DO Ó AND M. DE SouZA, A sharp inequality of Trudinger-Moser type and extremal functions in $H^{1, n}\left(\mathbb{R}^{n}\right)$, J. Differential Equations, 258, (2015) 4062-4101. 
[13] J. Do Ó AND M. DE SouZA, Trudinger-Moser inequality on the whole plane and extremal functions, Commun. Contemp. Math., 18, (2016) 1550054.

[14] Y. Fang And M. Zhang, On a class of Kazdan-Warner equations, Turkish J. Math., 42, (2018) 2400-2416.

[15] M. FluChER, Extremal functions for the trudinger-moser inequality in 2 dimensions, Comment. Math. Helv., 67, (1992) 471-497.

[16] M. GAZZini AND E. SERRA, The Neumann problem for the Hénon equation, trace inequalities and Steklov eigenvalues, Ann. Inst. H. Poincaré Anal. Non Linaire, 25, (2008) 281-302.

[17] S. IUlA AND G. MANCINI, Extremal functions for singular Moser-Trudinger embeddings, Nonlinear Anal., 156, (2017) 215-248.

[18] X. LI, An improved singular Trudinger-Moser inequality in $\mathbb{R}^{N}$ and its extremal functions, J. Math. Anal. Appl., 462, (2018) 1109-1129.

[19] X. Li AND Y. YANG, Extremal functions for singular Trudinger-Moser inequalities in the entire Euclidean space, J. Differential Equations, 264, (2018) 4901-4943.

[20] Y. LI, Moser-Trudinger inequality on compact Riemannian manifolds of dimension two, J. Partial Differential Equations, 14, (2001) 163-192.

[21] Y. LI, The existence of the extremal function of Moser-Trudinger inequality on compact Riemannian manifolds, Sci. China A, 48, (2005) 618-648.

[22] Y. Li AND B. RUF, A sharp Trudinger-Moser type inequality for unbounded domains in $R^{N}$, Ind. Univ. Math. J., 57, (2008) 451-480.

[23] K. Lin, Extremal functions for Moser's inequality, Trans. Amer. Math. Soc., 348, (1996) 2663-2671.

[24] P. L. Lions, The concentration-compactness principle in the calculus of variation, the limit case, part I, Rev. Mat. Iberoamericana, 1, (1985) 145-201.

[25] G. LU AND Y. YANG, The sharp constant and extremal functions for Moser-Trudinger inequalities involving $L^{p}$ norms, Discrete and Continuous Dynamical Systems, 25, (2009) 963-979.

[26] J. Moser, A sharp form of an inequality by N. Trudinger, Indiana Univ. Math. J., 20, (1970/71) $1077-1092$.

[27] V. NGUYEN, Improved Moser-Trudinger inequality for functions with mean value zero in $R^{n}$ and its extremal functions, Nonlinear Anal., 163, (2017) 127-145.

[28] V. NGUYEN, Improved Moser-Trudinger inequality of Tintarev type in dimension $n$ and the existence of its extremal functions, Ann. Glob. Anal. Geom., 54, (2018) 237-256.

[29] W. NI, A nonlinear Dirichlet problem on the unit ball and its applications, Indiana Univ. Math. J., 31, (1982) 801-807.

[30] J. PeETRE, Espaces d'interpolation et théorème de Soboleff, Ann. Inst. Fourier (Grenoble), 16, (1966) 279-317.

[31] S. Pohozaev, The Sobolev embedding in the special case $p l=n$, Proceedings of the technical scientific conference on advances of scientific reseach 1964-1965, Mathematics sections, Moscov. Energet. Inst., (1965) 158-170.

[32] P. TOLKSDORF, Regularity for a more general class of qusilinear elliptic equations, J. Differential Equations, 51, (1984) 126-150.

[33] N. Trudinger, On embeddings into Orlicz spaces and some applications, J. Math. Mech., 17, (1967) 473-484.

[34] Y. YANG, A sharp form of Moser-Trudinger inequality in high dimension, J. Funct. Anal., 239, (2006) $100-126$.

[35] Y. YANG, A sharp form of the Moser-Trudinger inequality on a compact Riemannian surface, Trans. Amer. Math. Soc., 359, (2007) 5761-5776.

[36] Y. YANG, Corrigendum to "A sharp form of Moser-Trudinger inequality in high dimension”, J. Funct. Anal., 242, (2007) 669-671.

[37] Y. YANG, Extremal functions for Trudinger-Moser inequalities of Adimurthi-Druet type in dimension two, J. Differential Equations, 258, (2015) 3161-3193.

[38] Y. YANG AND X. ZhU, Blow-up analysis concerning singular Trudinger-Moser inequalities in dimension two, J. Funct. Anal., 272, (2017) 3347-3374.

[39] Y. YANG AND X. ZHU, A Trudinger-Moser inequality for conical metric in the unit ball, Arch. Math. (Basel), 112, (2019) 531-545.

[40] V. I. YUdovich, Some estimates connected with integral operators and with solutions of elliptic equations, Dokl. Akad. Nauk SSSR, 138, (1961) 805-808. 
[41] J. ZHU, Improved Moser-Trudinger inequality involving $L^{p}$ norm in $n$ dimensions, Adv. Nonlinear Stud., 14, (2014) 273-293. 